\title{
Inheritance Arguments for Fundamentality
}

In R. Bliss \& G. Priest (eds.), Reality and its Structure: Essays in Fundamentality, Oxford University Press (forthcoming)

Kelly Trogdon

Abstract: According to metaphysical foundationalism, it's necessary that any nonfundamental entity be fully grounded by fundamental entities. Jonathan Schaffer has recently proposed an argument for metaphysical foundationalism that appeals to the notion of reality inheritance. After clarifying the argument, I argue that it is unsuccessful. Then I suggest that there are more plausible arguments that appeal to the notion of causal capacity inheritance instead. But, as these arguments target special cases of metaphysical foundationalism, I conclude that if considerations involving inheritance are to provide a route to metaphysical foundationalism, the route will be indirect.

\section{Introduction}

I'm a proponent of grounding-I think that the notion of grounding is coherent, figures in ordinary thinking, and deserves a place in our philosophical toolkit due to its theoretical utility. ${ }^{1}$ For the purposes of this chapter I assume that grounding is a relation between entities of various ontological categories and their categories needn't match. Where capital English letters take singular values and capital Greek letters plural values, grounding statements have the following form: $\Delta$ grounds $\mathrm{A} .^{2}$

It's a familiar idea that there must be a foundation for being. How can we sharpen up this idea? As is customary, let's say that an entity is fundamental just in case it isn't partially grounded. With recourse to fundamentality so understood we can put the idea as follows:

Metaphysical foundationalism: necessarily, any non-fundamental entity is fully grounded by fundamental entities.

Proponents of grounding tend to accept metaphysical foundationalism or similar theses. Here are three examples. First, Cameron (2008) argues that general considerations involving theoretical virtues suggest that metaphysical foundationalism without its modal preface is

\footnotetext{
${ }^{1}$ For introductions to and overviews of recent literature on grounding, see Bliss and Trogdon (2014), Clark and Liggins (2012), Correia and Schnieder (2012), Raven (2015), and Trogdon (2013).

2 See Schaffer 2009 for more on this view of the logical form of grounding statements. Audi (2012) and Rosen (2010) claim that the relata of the grounding relation instead are restricted to facts. I'm actually sympathetic with this view, but as Schaffer works with the category-neutral view and I'm going to engage with his argument for metaphysical foundationalism (a thesis I define shortly), I too will work with the category-neutral view.
} 
true. He writes, "If we seek to explain some phenomena, then, other things being equal, it is better to give the same explanation of each phenomenon than to give separate explanations of each phenomenon" and we can do that only if "every dependent entity has its ultimate ontological basis in some collection of independent entities" (12).

Second, Fine (2010) claims that metaphysical foundationalism is supported by the plausible idea that all non-fundamental entities have complete or satisfactory explanations. He writes, "But there is still a plausible demand on ground or explanation that we are unable to evade. For given a truth that stands in need of explanation, one naturally supposes that it should have a 'completely satisfactory' explanation, one that does not involve cycles and terminates in truths that do not stand in need of explanation" (105).

Third, Schaffer (2016), taking a page from Leibniz, argues that considerations involving the notion of reality inheritance support metaphysical foundationalism. Schaffer's argument in outline is this: since non-fundamental entities inherit their reality from what grounds them (equivalently: entities transfer reality to what they ground), it must be that non-fundamental entities are fully grounded by fundamental entities; otherwise, there would be no source of their reality, and the idea that there is reality inheritance in the absence of a source for reality is incoherent.

In this chapter I take up the question of how we might appeal to the notion of inheritance in arguing for metaphysical foundationalism. I first clarify Schaffer's inheritance argument sketched above, suggesting that it relies on a heavy-duty metaphysical principle I call the inheritance principle. I show that Schaffer's argument is unsuccessful even granting the principle. Then I explore what consequences the inheritance principle might have for metaphysical foundationalism granting for the sake of argument that the principle is true. I show how in this case we can deploy the notion of causal capacity inheritance in arguing for two special cases of metaphysical foundationalism, what I call causal foundationalism and concrete foundationalism. I conclude that if considerations involving inheritance are to provide a route to metaphysical foundationalism, the route will be indirect-in this case we would need to argue for the thesis in a piecemeal fashion (say, by arguing for concrete foundationalism plus a corresponding thesis about abstract entities). There may indeed be plausible inheritance arguments for fundamentality - it's just that we need to be careful to focus on the appropriate theses as well as the appropriate inherited properties.

\section{Reality inheritance}

An object is gunky just in case each of its proper parts has proper parts. Leibniz can be read as rejecting the possibility of gunk, as he claims that the reality of an aggregate is "borrowed" from its proper parts, and "... where there is no reality that is not borrowed, there will never be any reality, since it must belong ultimately to some subject” (Adams 1994, 335). In other 
words, every being of aggregation "... has its reality only from that of its components, so that it will have none at all if each being of which it is composed is again a being by aggregation..." (336). This line of thought plays a crucial part in Leibniz's argument for monads.

In a Leibnizian spirit, Schaffer articulates what I call the reality inheritance argument as follows:

... a grounded entity inherits its reality from its grounds, and where there is an inheritance there must be a source. One cannot be rich merely by having a limitless supply of debtors, each borrowing from the one before. There must actually be a source of money somewhere. Likewise, something cannot be real merely by having a limitless sequence of ancestors, each claiming reality from its parents. There must actually be a source of reality somewhere. Just as wealth endlessly borrowed is never achieved, so reality endlessly dependent is never realized. $(2016,95)$

Schaffer sees the primary conclusion of the argument as the claim that grounding is wellfounded in the sense that downwardly non-terminating chains of partial grounds are impossible. He claims that, were there such a grounding chain, the being of any object in that chain would be "infinitely deferred, never achieved" (2010, 62). Returning to Leibniz, he claims that in the event that every object is a being of aggregation "one never arrives at any real being" (Adams 1994, 333). ${ }^{3}$

If grounding is well-founded in Schaffer's sense it follows that metaphysical foundationalism as I've characterized it is true. But metaphysical foundationalism is compatible with downwardly non-terminating chains of partial grounds, so long as each link in such a chain is fully grounded by fundamental entities. Here's a potential example of such a grounding structure. Let $S_{1}$ be a spherical region of space, $S_{2}$ a proper sub-region of $S_{1}, S_{3}$ a proper subregion of $S_{2}$, and so on without end. You might claim that $S_{1}$ is partially grounded by $S_{2}, S_{2}$ is partially grounded by $S_{3}$, and so on without end. But suppose that there are spatial points. You might claim in this case that spatial points are fundamental, and each of the Ss is fully grounded by spatial points. ${ }^{4}$

Pace Schaffer, I think that the reality inheritance argument is better interpreted as an argument for metaphysical foundationalism as I have formulated the thesis than as an

\footnotetext{
3 While she doesn't discuss the reality inheritance argument, Bennett (2011) also claims that downwardly non-terminating chains of partial grounds are impossible.

${ }^{4}$ This case is adapted from cases discussed by Bliss (2013) and Rabin and Rabern (2016). See Dixon 2016 for potential examples of what he calls fully pedestalled chains, grounding structures that contain downwardly non-terminating chains of partial grounds each of whose links is fully grounded by the same fundamental entities. In the example above, each region in the downwardly non-terminating chain is grounded by different (but overlapping) collections of points. Rabin and Rabern's (2016) version of the region/point example is intended to be an example of a fully pedestalled chain.
} 
argument for the claim that downwardly non-terminating chains of partial grounds are impossible. This is for the simple reason that the argument seems to proceed upon the idea that there is something wrong about inheritance in the absence of a source, not that there is something wrong about endless inheritance per se. Returning to the region/point case, this grounding structure isn't objectionable so far as the reality inheritance argument is concerned. It's true that the spherical region is real by way of endless reality inheritance, but there is nonetheless a source of its reality — the points that it contains. While their focus isn't the reality inheritance argument, Rabin and Rabern make essentially the same point: "Only when there is no origin for being, no fundamental substratum to the hierarchy of ground, is... being 'infinitely deferred and never achieved'. In [cases like the region/point case] being is infinitely deferred but nevertheless achieved" (2016, 363).

Provided that the conclusion of the reality inheritance argument is that metaphysical foundationalism is true, I see the argument as consisting of the following three theses:

The reality inheritance premise: necessarily, if A is non-fundamental then A inherits its reality from whatever fully grounds it.

The source of reality premise: necessarily, if $\mathrm{A}$ inherits its reality then there is some $\Delta$ that is a source of A's reality (i.e. A inherits its reality from $\Delta$ and no entity among $\Delta$ inherits its reality).

The reality/fundamentality premise: necessarily, if $\Delta$ is a source of A's reality then the entities among $\Delta$ are fundamental and $\Delta$ fully grounds $\mathrm{A}$.

Two points of clarification are as follows. First, I assume that talk of reality and talk of existence in this context come to the same thing-for something to be real is just for it to exist. Second, a background assumption of the argument is that reality (existence) is a property - the book on the table, for example, has the property being real. The reality inheritance argument appeals to the idea that being real is transferable-when $\Delta$ fully grounds A the latter inherits this property from the former. ${ }^{5}$

Metaphysical foundationalism follows from the premises of the reality inheritance argument. What can be said on behalf of each premise? I begin with the reality inheritance premise: necessarily, if $\mathrm{A}$ is non-fundamental then $\mathrm{A}$ inherits its reality from whatever fully grounds it. I take it that for Schaffer it's a stipulative matter that $\Delta$ fully grounds $A$ just in case the latter inherits its reality from the former. Viewed in this way, the reality inheritance premise isn't

\footnotetext{
${ }^{5}$ Here we can follow Salmon (1987) and claim that the property expressed by 'exists' and 'is real' is the property being identical to sometbing or other, and an entity has this property just in case it's not the case that everything is distinct from it. For a different conception of reality-one whereby existence and reality can come apart—see Fine 2001.
} 
something that really stands in need of argument, as it's a consequence of the particular conception of grounding that Schaffer has in mind.

Now I turn to the source of reality premise: necessarily, if $\mathrm{A}$ inherits its reality then there is some $\Delta$ that is a source of A's reality (i.e. A inherits its reality from $\Delta$, and no entity among $\Delta$ inherits its reality). I take it that the rationale for this premise doesn't proceed upon considerations about grounding, explanation, or reality inheritance per se. Instead, it appeals to general considerations about inheritance. In particular, I see the rationale for the source of reality premise as appealing to the following principle, where lower case Greek letters range of properties:

The inheritance principle: necessarily, if $\mathrm{A}$ inherits $\varphi$ then there is some $\Delta$ that is a source of A's $\varphi$-ness (i.e. A inherits $\varphi$ from $\Delta$ and no entity among $\Delta$ inherits $\varphi$ ).

To motivate the inheritance principle you might begin by claiming that there are ordinary properties that seem to conform to it, where $\varphi$ conforms to the inheritance principle just in case $\varphi$ is inheritable and it's necessary that something inherits $\varphi$ only if there is a source of that entity's $\varphi$-ness. Schaffer's example is wealth. For another example, consider the property being royal. This property is inheritable, and it seems necessary that people are royal only if there is a source for their royalty. As Brzozowski puts the idea, in the absence of a source for royalty no one is royal, for in this case "...there is nothing in the world that makes it the case that someone is royal in the first place...” $(2008,200)$.

You might also claim that regresses that violate the inheritance principle seem vicious. Consider the following version of Bradley's regress. Suppose that a has F. So the fact that a is F obtains. A fact obtains only if its constituents are unified or connected. Let $U$ be the property of having unified constituents, and I the instantiation relation. So the fact that a is F has $\mathrm{U}$. The fact that a is $\mathrm{F}$ inherits its U-ness from a further fact- the fact that a stands in I to F. As you must have $\mathrm{U}$ to transfer $\mathrm{U}$-ness, the latter fact also has $\mathrm{U}$. The fact that a stands in I to F inherits its $\mathrm{U}$-ness from another fact-the fact that $<\mathrm{a}, \mathrm{F}>$ stands in I to I. As you must have $U$ to transfer U-ness, the latter fact also has $U$. And the fact that $<a, F>$ stands in I to I inherits its U-ness from another fact-the fact that $<<a, F>, I>$ stands in I to I. This goes on without end. This regress violates the inheritance principle-each fact in the series inherits its $\mathrm{U}$-ness, and for any fact in the series there is no source for its U-ness (i.e. anything from which it inherits its U-ness has its own U-ness by way of inheritance). And many have claimed that the regress interpreted along these lines is vicious. ${ }^{6}$

${ }^{6}$ There are two ways for a regress not to violate the inheritance principle. First, it might not involve transference. Consider the benign regress of natural numbers. Why do we think that there is such a regress? Well, we don't think so because we think that the property having a successor is an inheritable feature, something transferred from one natural number to another. Indeed, this regress doesn't seem 
Finally, I turn to the reality/fundamentality premise: necessarily, if $\Delta$ is a source of A's reality then the entities among $\Delta$ are fundamental and $\Delta$ fully grounds A. Suppose that $\Delta$ is a source of A's reality. Hence, (i) A inherits its reality from $\Delta$ and (ii) no entity among $\Delta$ is real by way of reality inheritance. Given (i) and the stipulation that some entities fully ground another just in case the latter inherits its reality from the former, $\mathrm{A}$ is fully grounded by $\Delta$. Given (ii) and the reality inheritance premise (which itself follows from the stipulation about grounding), the entities among $\Delta$ are fundamental. ${ }^{7}$

\section{A problem for the reality inheritance argument}

Should we accept metaphysical foundationalism on the basis of the reality inheritance argument? Morganti (2015) is unconvinced-he questions in particular the reality inheritance premise. He suggests that, rather than entities transferring reality to what they ground, it may be that reality emerges from grounding chains instead. What Morganti says here is modeled after what Klein (2007) says in defense of epistemic infinitism. Many claim that epistemic properties are inheritable. It's a familiar idea that there are conditions under which knowledge is transferred via testimony from one person to another and justification is transferred via inferential connections from one of your beliefs to another. Klein claims, however, that justification (and presumably knowledge) isn't a transferable property. Instead, we should think of justification "as emerging whenever there is an endless, non-repeating set of propositions available as reasons” $(2007,16)$.

Suppose that the relevant notion of reality emergence is consistent, as Morganti argues. Remember, however, that the reality inheritance premise is plausibly viewed as a consequence of a stipulation about grounding. So the proponent of the reality inheritance argument might respond that, while the notion of grounding*_a notion characterized partly

to involve the transference of any particular property. Instead, we think that there is an infinite regress of natural numbers because we accept Peano's axioms of arithmetic, and it follows from them that there is such a regress. Second, the regress might involve the transference of $\varphi$ where, for any inheritor of $\varphi$ in that regress, there is a source of that entity's $\varphi$-ness. Consider, for example, Tarski's benign truth regress: supposing that sentence $\mathrm{S}$ is true, ' $\mathrm{S}$ is true' is true; " $\mathrm{S}$ is true' is true' is true; and so on without end. While ' $\mathrm{S}$ is true' inherits its truth from $\mathrm{S}$, the latter is a source of the former's truth. The same applies to the iterated sentences.

7 While Schaffer's reality inheritance argument is Leibnizian in character, you might think that its historical roots trace further back, at least as far as Aquinas's Five Ways. I read Aquinas, however, as rejecting the inheritance principle. Consider, for example, a chain of royalty inheritance in the absence of a source for royalty, what Aquinas would call a causal series ordered per accidens without a first cause. Apparently Aquinas thinks that such a causal series is possible but argues that there is another type of causal series - a causal series ordered per se- that must have first causes (see Davies 2014, Ch. 3). If this is right then the standard responses to Aquinas's arguments concerning first causes don't transfer in a straightforward manner to the reality inheritance argument. Thanks to Einar Bohn for helpful discussion here. 
in terms of the notion of reality emergence rather than that of reality inheritance-is consistent and perhaps worth exploring further, it simply isn't the relevant concept here. Metaphysical foundationalism and the reality inheritance argument appeal to the notion of grounding rather than grounding*, so to take up the latter in this context is just to change the subject. ${ }^{8}$

The source of reality premise is the most substantive premise of the reality inheritance argument. So the most obvious strategy to pursue in responding to the argument is this: grant that the inheritance principle, if true, secures the source of reality premise, but reject this heavy-duty metaphysical principle. Bliss (2013), for example, considers a principle similar in spirit to the inheritance principle-roughly, it's necessary that if something has $\varphi$ conditionally then there is something that has $\varphi$ categorically-and argues that the principle is unmotivated.

Another critical approach to the inheritance principle is this: grant that the principle seems plausible on first inspection but claim that this appearance of plausibility can be explained away. Here's one proposal along these lines. As a matter of fact, whenever A has $\varphi$ by way of diachronic inheritance-inheritance of $\varphi$ that unfolds over time- the inheritor is such that there is a source of its $\varphi$-ness. But this is simply because the universe as a matter of fact is temporally finite. As the universe has a beginning, the chain of diachronic inheritance by which A has $\varphi$ also has a beginning. There are possible worlds, however, in which the universe is temporally infinite. In some of these worlds Bill's wealth, for example, was inherited from Bob, Bob's wealth was inherited from Bert, and so on, without anyone having amassed wealth by honest work in the first place. What has happened here is that we've mistaken a contingent fact - the fact that if A has $\varphi$ by way of diachronic inheritance then there is a source of A's $\varphi$-ness-for a necessary fact, one flowing from the nature of the inheritance relation, of which diachronic and synchronic inheritance (the type of inheritance at issue with grounding) are species. But in reality neither diachronic inheritance nor synchronic inheritance is such that where there is inheritance of some feature there must be a source of that feature. This explains why we find the inheritance principle plausible when in fact it's false. ${ }^{9}$

8 To be fair, however, Morganti's (2015) ultimate goal is to develop an alternative to metaphysical foundationalism - what he calls metaphysical infinitism - rather than simply object to the reality inheritance argument. And I take it that Morganti would reject the idea that he and Schaffer are simply talking past each other and claim instead that Schaffer is just wrong to claim that grounding involves reality inheritance.

${ }^{9}$ Thanks to Ben Jantzen for helpful discussion here. 
Rather than argue that the inheritance principle is false, I'm going to approach the reality inheritance argument differently-I'll show that the source of reality premise is either unmotivated or false even granting the inheritance principle. The problem can be stated in terms of a dilemma. Either the relevant notion of reality inheritance is primitive or it's molecular in nature, consisting of the notion of reality and that of inheritance. If the former holds then the source of reality premise is unmotivated, and if the latter holds the premise (as well as the reality inheritance and reality/fundamentality premises) is false. And this holds despite the fact that the inheritance principle (we will assume) is true.

Taking the first horn, suppose that the notion of reality inheritance at issue in the reality inheritance argument is primitive in nature. As Schaffer's comparison between reality and wealth above indicates, the rationale for the source of reality premise is supposed to proceed upon general considerations about inheritance. In particular, it seems that the rationale appeals to the inheritance principle, as I noted above. But if the notion of reality inheritance is primitive, it's unclear why considerations regarding the general notion of inheritance should be relevant here-despite the common terminology, the notion of reality inheritance and that of inheritance per se might have nothing to do with each other! Conclusion: in this case the source of reality premise is unmotivated.

The point here isn't that there can't be interesting connections between the (presumably) primitive notion of grounding and other conceptual primitives, as there can be such connections. The point instead is that if the relevant notion of reality inheritance is primitive then it's unclear just what the concept comes to. In this case it's unclear just what theoretical payoff there would be to claiming that grounding involves reality inheritance.

Interestingly there are other places in the literature on grounding in which similar reasoning applies. Dasgupta (2014), Fine (2012), and Litland (2015) identify grounding with what they call metaphysical explanation. And they claim that by reflecting on the nature of explanation per se we can draw conclusions about grounding. For example, Fine (2010) suggests that since explanation is asymmetric, grounding is asymmetric as well. And Dasgupta (2014) argues that, as any part of an explanation is relevant to what it explains, any part of a ground is relevant to what it grounds. But note that if the notion of metaphysical explanation is primitive, it's unclear why considerations regarding explanation should be relevant heredespite the common terminology, the notion of metaphysical explanation and that of explanation per se might have nothing to do with each other! $!^{10}$

\footnotetext{
10 Thanks to an anonymous referee for suggesting that a comparison to discussions about the connection between grounding and explanation might be useful. I think that there is actually a problem for Dasgupta et al. here. They claim that grounding is a primitive notion. But if grounding is primitive then when they claim that grounding just is metaphysical explanation they must be taking the notion of metaphysical explanation as primitive as well. But if the relevant notion of metaphysical
} 
I now turn to the second horn, which is a bit more complicated. To begin, as Bennett (2011) and others note, grounding is generative. As we will understand the notion here, a relation is generative just in case its instantiation brings things into existence. ${ }^{11}$ Grounding is generative given that grounded entities exist due to grounding. Non-Humeans about causation think that the same is true of causation-caused events exist or occur due to causation. The relation of being-taller-than, by contrast, is non-generative-supposing that Sally is taller than Barbara, neither Sally nor Barbara exists due to being-taller-than. It seems that the relation of inheritance is also non-generative-it's never the case that an inheritor exists due to inheritance. It's an inheritor due to inheritance, but it exists for some other reason, if for any reason at all. In other words, it's never the case that a transferee-something to which a property is transferred-exists due to transference. Again, it's a transferee due to transference, but it exists for some other reason, if any reason at all. ${ }^{12}$

Generative relations, as I understand them, are 'full-time' generators - their instances always generate. But might there also be relations that, while not full-time generators, are 'part-time' generators, relations such that, while some of their instances don't involve generation, others do? You might think that transference in particular is such a relation. It's true that transference isn't a full-time generator-when, say, wealth is transferred the existence of the transferee isn't due to this instance of transference. But its existence nevertheless might be due to another instance of transference. The idea is that when being real instead is transferred, the existence of the transferee is indeed due to this instance of transference.

I have no problem with the distinction between full- and part-time generators - the notion of a part-time generator seems perfectly coherent. Instead, the ultimate problem as I see it is this: the idea that the property being real in particular is transferrable just doesn't make sense. If something exists by way of the transference of being real then the entity's status as a feature recipient is explanatorily relevant to its existence. But it seems that the explanation goes in the other direction: the fact that the entity exists instead is explanatorily relevant to its status as a feature recipient. ${ }^{13}$

explanation is primitive it's unclear that it's appropriate to draw lessons about the nature of grounding from considerations about explanation per se.

11 See Bennett 2017, Ch. 3 for further discussion of the relevant notion of generation.

12 I should also note that the relevant notion of inheritance here is quasi-technical-while there are similarities between its conditions of application and those of the ordinary notion(s) of inheritance, there may be differences as well. Example: while the quasi-technical notion is such that synchronic inheritance is possible, perhaps the ordinary conception is such that all inheritance is diachronic. So what I'm claiming above in effect is this: one crucial similarity between ordinary notion(s) and the relevant quasi-technical notion of inheritance is that inheritance is non-generative in the sense that inheritors don't exist due to inheritance.

${ }^{13}$ Thanks to Cruz Davis and two anonymous referees for helpful discussion here. 
Returning to the second horn, now suppose that the relevant notion of reality inheritance in the reality inheritance argument is molecular, consisting of the notion of reality and that of inheritance per se. The notion of reality inheritance in this case is inconsistent, as the rules governing its use themselves are inconsistent. On the one hand, if we judge that an inheritor exists due to inheritance then we're using the notion of inheritance incorrectly, as I pointed out above. And, as this concept is a component of the notion of reality inheritance, the same applies to the molecular concept - if we judge that an inheritor exists due to reality inheritance (or any other type of inheritance) then we're likewise using the notion of reality inheritance incorrectly. (Compare: supposing that if we judge that cows are numbers we're using the notion of cows incorrectly, it follows that if we judge that brown cows are numbers we're using the notion of brown cows incorrectly as well, on the assumption that the latter is a molecular concept consisting of the notion of the color brown and that of cows.) On the other hand, the application conditions for the molecular concept are such that it applies when an inheritor exists due to reality inheritance. Hence, the notion of reality inheritance is inconsistent. Conclusion: in this case the source of reality premise (as well as the reality inheritance and the reality/fundamentality premises) is false as it's semantically defective.

\section{Causal capacity inheritance}

Above I argued that the reality inheritance argument fails even granting the inheritance principle. In this section I argue that this principle nevertheless may have important consequences for metaphysical foundationalism. I show in particular that if we grant the inheritance principle and focus on the appropriate inheritable property, it may be that we have the resources to establish special cases of metaphysical foundationalism. This is good news for those both sympathetic with the inheritance principle and the idea that there are fundamental entities. And, even if you're skeptical of the inheritance principle, it's still worth knowing what its consequences for fundamentality might be, were the principle true.

If we're going to rely on the inheritance principle then the property being real isn't the property that we should focus on, given what I argued above. In this case what property should we target? I propose having the capacity for causal activity (causal capacity for short). An entity has this property just in case it has causal powers, dispositions to enter into particular sorts of causal transactions. To specify the causal powers of an entity is to specify just which sorts of effects that entity is apt to causally contribute to. What sorts of entities can have causal powers? I assume that property instances are the primary bearers of causal powers. Property instances are like ordinary objects in that they're concrete, but they're like properties in that they 'go with' entities. Following Lowe (2006, Ch. 2), a property goes with an entity when the latter instantiates the former and a property instance does so when it characterizes that entity. As Pereboom puts the idea, property instances are "concrete ways particular things are" $(2011,141)$. Consider, for example, the property being magnetic. 
Instances of this property both characterize certain objects (magnets) and are apt to enter into particular sorts of causal transactions (e.g. attracting nearby pins).

Suppose that an instance of $\varphi$ inherits its causal capacity from an instance of $\psi$. There are different ways of thinking about just how the causal powers of these property instances are related in this case. Following Shoemaker (2007, Ch. 1), you might claim that, for any instance of a causal power that characterizes the $\varphi$-instance, there is some instance of a causal power that characterizes the $\psi$-instance such that the former is identical to the latter. Or, following Pereboom (2011, Ch. 7) you might instead claim that any instance of a causal power that characterizes the $\varphi$-instance is constituted by instances of causal powers that characterize the $\psi$-instance. I won't take a stand on the matter here. ${ }^{14}$

With the notion of causal capacity inheritance on the table, consider the following special case of metaphysical foundationalism:

Causal foundationalism: necessarily, any non-fundamental entity with causal capacity is fully grounded by fundamental entities.

If metaphysical foundationalism is true then so too is causal foundationalism. But the truth of the latter is compatible with the falsity of the former-while it may be necessary that any non-fundamental entity with causal capacity is fully grounded by fundamental entities, perhaps it's not necessary that any non-fundamental entity without causal capacity is so grounded. Putting aside metaphysical foundationalism for the moment, what reason, if any, do we have to think that causal foundationalism is true? Here is an argument for the thesis that appeals to causal capacity inheritance:

The causal inheritance premise: necessarily, if A is non-fundamental and has causal capacity then A inherits its causal capacity from whatever fully grounds it.

The source of causal capacity premise: necessarily, if A inherits its causal capacity then there is some $\Delta$ that is a source of A's causal capacity (i.e. A inherits its causal capacity from $\Delta$ and no entity among $\Delta$ inherits its causal capacity).

The causality/fundamentality premise: necessarily, if $\Delta$ is a source of A's causal capacity then the entities among $\Delta$ are fundamental and $\Delta$ fully grounds $A$.

\footnotetext{
${ }^{14}$ Whatever we end up saying here, presumably it should be compatible with the idea that some property instances have type-distinct causal powers from the causal powers of the property instances from which they inherit their causal capacity. The Pereboom-inspired proposal apparently has this feature. It may be, however, that the Shoemaker-inspired proposal doesn't have this feature, given reasonable assumptions about property individuation.
} 
Causal foundationalism follows from these premises. What can be said on behalf of each premise of the argument? I begin with the causal inheritance premise. The motivation for this premise as I see it is this: it plays an essential role in a plausible response to a generalized version of Kim's (2007, Ch. 1) causal exclusion argument, an argument that challenges the very idea that non-fundamental property instances can enter into causal relations. ${ }^{15}$

The argument I have in mind appeals to two substantive principles, the first of which is Kim's causal exclusion principle. There are various formulations of this principle, but for our purposes the following formulation will do: no property instance has simultaneous full causes. ${ }^{16}$ The second principle is a grounding analogue to the familiar principle of physical causal closure, where the latter says that if a property instance has a full cause then it has a simultaneous full physical cause. The grounding analogue - the causal closure of grounding — is (roughly) this: if a property instance has a full non-fundamental cause then whatever fully grounds that cause is also a full cause of the property instance. Turning to the argument, suppose, for reductio, that an instance of $\varphi$ is both non-fundamental and fully causes an instance of property $\psi$. By the causal closure of grounding, there is something - a property instance - that fully grounds the $\varphi$-instance and fully causes the $\psi$-instance. As grounding is irreflexive, this something and the $\varphi$-instance are distinct. Given that grounding is synchronic, the $\psi$-instance has two simultaneous full causes. By the causal exclusion principle, no event has two simultaneous full causes. By reductio, it's not the case that there is an instance of $\varphi$ that is both non-fundamental and fully causes an instance of $\psi$.

A plausible response to this argument-versions of which have been proposed as responses to Kim's original version of argument - is that there are exceptions to the causal exclusion principle when causal capacity inheritance is involved. The idea in essence is that property instances can have simultaneous full causes so long as one of the causes inherits its causal capacity from the other. The intuition behind this proposal is that where there is causal capacity inheritance there isn't causal competition and the exclusion principle applies only when there is causal competition. Provided that the causal inheritance premise-necessarily, if $\mathrm{A}$ is non-fundamental and has causal capacity then A inherits its causal capacity from whatever fully grounds it-is true, we can avail ourselves of this response.

Now, the issues here are obviously complex and controversial. Just what we should ultimately think about all of this isn't something that we need to decide here. For my

\footnotetext{
${ }^{15}$ Kim speaks of inheritance in his work on realization, but he focuses on the inheritance of particular causal powers rather than causal capacity in general. He claims, for example, that "...higher states... inherit their causal powers from the underlying states that realize them" (1993, 335).

16 The argument can be recast with a weaker formulation of the causal exclusion principle-one that merely rules out systematic causal overdetermination-but I won't take up that version of the argument here.
} 
purposes, the following modest claim is enough: issues concerning causal exclusion suggest that the causal inheritance premise is worth taking seriously.

Next, the source of causal capacity premise: necessarily, if A inherits its causal capacity then there is some $\Delta$ that is a source of A's causal capacity (i.e. A inherits its causal capacity from $\Delta$ and no entity among $\Delta$ inherits its causal capacity). This premise is motivated by the inheritance principle, which we're assuming for the sake of argument is true. A crucial difference between the source of causal capacity premise and the source of reality premise, then, is this: while the inheritance principle doesn't support the latter, it does support the former.

Finally, the causality/fundamentality premise: necessarily, if $\Delta$ is a source of A's causal capacity then the entities among $\Delta$ are fundamental and $\Delta$ fully grounds $A$. Suppose that $\Delta$ is a source of A's causal capacity. Hence, (i) A inherits its causal capacity from $\Delta$, and (ii) none of the entities among $\Delta$ have their causal capacity by way of inheritance. Given (ii) and the causal inheritance premise, the entities among $\Delta$ are fundamental. Why think, however, that A is fully grounded by $\Delta$ in this case? Given (i) we get this result if the following thesis is true: it's necessary that if A inherits its causal capacity from $\Delta$ then the latter fully grounds the former. What reason, if any, do we have for thinking that this additional thesis is true?

Let's start with a mundane observation: there are different ways of traveling from one town to another that require distinctive sorts of links between the towns. Travel by car, for example, requires that roads connect the towns, while travel by foot requires that footpaths, sidewalks, and so on, connect them. Similarly, there are different inheritable properties whose transference between entities requires distinctive sorts of links between them. Returning to two examples mentioned above, the transference of knowledge from one individual to another requires that testimony link those individuals (it must be that one testifies on some matter to the other), and the transference of justification from one of your beliefs to another requires there to be an inferential link between your beliefs. Returning to the additional thesis about the connection between causal capacity inheritance and grounding set out above, the idea is that grounding is the distinctive sort of link between property instances that the transference of causal capacity requires.

What else can be said on behalf of the additional thesis? Well, paradigmatic cases of causal capacity inheritance are such that inheritors are fully grounded by the entities from which they inherit, and this gives us reason to take seriously the idea that it's necessary that if A inherits its causal capacity from $\Delta$ then the latter fully grounds the former. I'll consider three sorts of cases. In the first case some of the relevant property instances characterize the same entity, in the second some characterize distinct but materially coincident entities, and in the third some characterize objects at different levels of mereological aggregation. 
First, suppose that various DNA molecules are embedded in a chemical system in such a way that they play the causal role characteristic of genes. In this case, an instance of the property of being a gene inherits its capacity for causal activity from instances of various properties concerning these molecules and the system in which they're embedded. And the instance of being a gene is fully grounded by these property instances. Second, suppose that people are related to a lump of clay on a pedestal in such a way that there is a statue coincident with it. In this case, an instance of the property being a statue inherits its capacity for causal activity from instances of various properties concerning the clay and the people who are relevantly related to the clay. And the instance of being a statue is fully grounded by these property instances. Third, suppose that there are carbon molecules arranged in such a way that there is a diamond. In this case, an instance of the property being a diamond inherits its causal capacity from instances of various properties concerning the molecules that compose the diamond and their arrangement. And the instance of being a diamond is fully grounded by these property instances.

I've shown how we can appeal to the notion of causal capacity inheritance in arguing for causal foundationalism. The notion can also be deployed in defending another special case of metaphysical foundationalism:

Concrete foundationalism: necessarily, any non-fundamental concrete entity is fully grounded by fundamental concrete entities. ${ }^{17}$

Consider the following familiar thesis:

The concrete principle: necessarily, an entity is concrete just in case it has causal capacity. Note that the considerations I outlined above that support causal foundationalism also support the following thesis: necessarily, any entity with causal capacity is fully grounded by fundamental entities with causal capacity. If this thesis and the concrete principle are true, concrete foundationalism follows.

Three additional points worth noting that go beyond causal and concrete foundationalism are these. First, note that from the causal inheritance premise it follows that no non-concrete entity can ground a concrete entity, provided that the concrete principle is true. Since nonconcrete entities lack causal capacity, they can't transfer this capacity to entities they ground.

\footnotetext{
${ }^{17}$ In defending priority monism, Schaffer's (2010) discussion of metaphysical foundationalism is restricted to concrete entities - the operative principle in his discussion is this: any concrete entity that is grounded by concreta is ultimately grounded by concreta that aren't themselves grounded by concreta. See Trogdon 2017 for related discussion.
} 
In this case, if 'concrete' and 'abstract' are contraries (so any abstract entity is non-concrete), it follows that abstract entities can't ground concrete entities. ${ }^{18}$

The second point concerns the neo-Aristotelian view according to which properties, while abstract, are fully grounded by concrete entities. Consider the following thesis: necessarily, if an entity is fully grounded by entities with causal capacity then the former inherits this capacity from the latter. This thesis is related to the causal inheritance premise-while neither thesis entails the other, they form a natural package. If this thesis is true then the neo-Aristotelian view is false, provided that the concrete premise is true and any abstract entity is non-concrete.

The third point concerns the connection between causal capacity and reality. Armstrong (1978, Ch. 12, 16) and others endorse eleaticism, which I understand to be the view that the property of existing and the property of having causal capacity are the same property. Now, the discussion above indicates that the property of having causal capacity is an inheritable feature. Hence, eleaticism has the consequence that reality is an inheritable feature, as the property of existing and the property of being real are the same property. But I've argued that reality isn't an inheritable feature. If I'm right about that, it follows that eleaticism is false.

\section{Conclusion}

I've argued that it's more fruitful to focus on causal capacity inheritance than reality inheritance in arguing for foundationalist theses in metaphysics. First I argued that Schaffer's reality inheritance argument for metaphysical foundationalism is unsuccessful even granting the inheritance principle. Then I showed how to appeal to this principle and the notion of causal capacity inheritance in arguing for special cases of metaphysical foundationalism. What, however, about the general thesis of metaphysical foundationalism?

Consider the following thesis:

Abstract foundationalism: necessarily, any non-fundamental abstract entity is fully grounded by fundamental abstract entities. ${ }^{19}$

Supposing, pace Williamson (2013, Ch. 1) and others that 'concrete' and 'abstract' are contradictories (so it's necessary that any entity be concrete or abstract), if both concrete and

\footnotetext{
${ }^{18}$ For an alternative view, see Carmichael 2016 for an argument that facts about concrete objects are grounded by facts about abstract objects.

${ }^{19}$ See Trogdon and Cowling (manuscript) for further discussion of metaphysical foundationalism restricted to abstract entities - the operative principle in their discussion is this: any abstract entity that is grounded by abstracta is ultimately grounded by abstracta that aren't themselves grounded by abstracta.
} 
abstract foundationalism are true it follows that metaphysical foundationalism is also true. I've argued that concrete foundationalism is worth taking seriously provided that the inheritance principle is true. What about abstract foundationalism?

I'm not going to provide an argument for the view here. Instead, I'll conclude with a recipe for constructing such an argument. Recall the concrete principle discussed above. It identifies a positive theoretical role for the notion of being concrete to play-concrete entities are those that have the capacity for causal activity. And as I argued above, the property at issue here-having the capacity for causal activity - is inheritable. So one way to argue for abstract foundationalism is to identify a positive theoretical role for the notion of being abstract to play, extract an inheritable property from this role, and then provide an inheritance-style argument for abstract foundationalism in terms of this property. Such an argument would be structurally similar to the one I provided for causal foundationalism. Whether a plausible argument for abstract foundationalism along these lines can be given is something worth further thought. ${ }^{20}$

Is metaphysical foundationalism plausible? The verdict, so far as inheritance arguments are concerned, is still out. It seems fairly clear, however, that if considerations involving inheritance are to provide a route to metaphysical foundationalism, the route will be indirect—we would need to argue for the thesis in a piecemeal fashion. ${ }^{21}$

\section{References}

Adams, R.M. 1994. Leibniz: Determinist, Theist, Idealist. Oxford University Press.

Armstrong, D.M. 1978. Universals and Scientific Realism, Vols. 1 \& 2. Cambridge University Press.

Audi, P. 2012. "Grounding: Toward a Theory of the In-Virtue-Of Relation,” Journal of Philosophy 109: 685-711.

Bennett, K. 2011. “By Our Bootstraps,” Philosophical Perspectives 25: 27-41.

Bennett, K. 2017. Making Things Up. Oxford University Press.

\footnotetext{
${ }^{20}$ One complication: Williamson (2013, Ch. 1) argues that there being a positive theoretical role for the notion of being abstract to play counts against the idea that 'concrete' and 'abstract' are contradictories.

21 Thanks to my audience at the Canadian Philosophical Association conference at the University of Ottawa (6/1/15) for their comments and suggestions, particularly Sam Cowling, Michaela

McSweeney, and Joshua Spencer. Thanks also to Einar Bohn, Cruz Davis, Ben Jantzen, Ted Parent, and two anonymous referees for helpful comments.
} 
Bliss, R. 2013. "Viciousness and the Structure of Reality," Philosophical Studies 166: 399-418.

Bliss, R., and Trogdon, K. 2014. “Metaphysical Grounding.” In E. Zalta (ed.), The Stanford Encyclopedia of Philosophy.

https://plato.stanford.edu/archives/win2016/entries/grounding/

Brzozowski, J. 2008. “On Locating Composite Objects.” In D. Zimmerman (ed.), Oxford Studies in Metaphysics, Vol. 4. Oxford University Press.

Cameron, R. 2008. "Turtles All the Way Down: Regress, Priority and Fundamentality," Philosophical Quarterly 58: 1-14.

Carmichael, C. 2016. “Deep Platonism,” Philosophy and Phenomenological Research 92: 307-328.

Clark, M., and Liggins, D. 2012. “Recent Work on Grounding,” Analysis 72: 812-823.

Correia, F., and Schnieder, B. 2012. "Grounding: An Opinioned Introduction.” In F. Correia and B. Schnieder (eds.), Metaphysical Grounding: Understanding the Structure of Reality. Cambridge University Press.

Dasgupta, S. 2014. “On the Plurality of Grounds,” Philosophers' Imprint 14: 1-28.

Davies, B. 2014. Thomas Aquinas's Summa Theologiae: A Guide and Commentary. Oxford University Press.

Dixon, S. 2016. "What Is the Well-Foundedness of Grounding?” Mind 125: 439-68.

Fine, K. 2001. “The Question of Realism,” Philosophers' Imprint 1: 1-30.

Fine, K. 2010. “Some Puzzles of Ground,” Notre Dame Journal of Formal Logic 51: 97-118.

Fine, K. 2012. “A Guide to Ground.” In F. Correia and B. Schnieder (eds.), Metaphysical Grounding: Understanding the Structure of Reality. Cambridge University Press.

Kim, J. 1993. “The Nonreductivist's Troubles with Mental Causation.” Reprinted in Kim's Supervenience and Mind (1993). Cambridge University Press.

Kim, J. 2007. Physicalism or Something Near Enough. Princeton University Press.

Klein, P. 2007. "Human Knowledge and the Infinite Progress of Reasoning," Philosophical Studies 134: 1-17.

Litland, J. 2015. "Grounding, Explanation, and the Limit of Internality," Philosophical Review 124: 481-532. 
Lowe, E. J. 2006. The Four-Category Ontology: A Metaphysical Foundation for Natural Science. Oxford University Press.

Morganti, M. 2015. "Dependence, Justification and Explanation: Must Reality Be WellFounded?” Erkenntnis 80: 555-572.

Pereboom, D. 2011. Consciousness and the Prospects for Physicalism. Oxford University Press.

Rabin, G. O. and B. Rabern. 2016. "Well Founding Grounding Grounding," Journal of Philosophical Logic 45: 349-79.

Raven, M. 2015. “Ground,” Philosophical Compass 10: 322-333.

Rosen, G. 2010. “Metaphysical Dependence: Grounding and Reduction.” In R. Hale and A. Hoffman (eds.), Modality: Metaphysics, Logic, and Epistemology. Oxford University Press.

Salmon, N. 1987. "Existence." Reprinted in Metaphysics, Mathematics, and Meaning. Oxford University Press, 2005.

Schaffer, J. 2009. “On What Grounds What.” In D. Chalmers, D. Manley, and R. Wasserman (eds.), Metametaphysics. Oxford University Press.

Schaffer, J. 2010. “Monism: The Priority of the Whole,” Philosophical Review 119: 31-76.

Schaffer, J.2016. "Grounding on the Image of Causation,” Philosophical Studies 173: 49-100.

Shoemaker, S. 2007. Physical Realization. Oxford University Press.

Trogdon, K. 2013. “An Introduction to Grounding.” In M. Hoeltje, B. Schnieder, \& A. Steinberg (eds.), V arieties of Dependence. Philosophia Verlag.

Trogdon, K. 2017. "Priority Monism,” Philosophy Compass 12: 1-10.

Trogdon, K. and S. Cowling. Manuscript. "Prioritizing Platonism."

Williamson, T. 2013. Modal Logic as Metaphysics. Oxford University Press. 